\title{
CONSTRUCCIÓN DE UN INDICADOR DE POBREZA MULTIDIMENSIONAL MEDIANTE ANÁLISIS COMBINATORIO
}

Gary Canaviri-Sillerico Francisco Javier Aliaga-Lordemann 
Panorama Económico, 24 (Octubre 2016 - Septiembre 2017), pp. 85-104

Gary Canaviri-Sillerico

Francisco Javier Aliaga-Lordemann

\title{
Construcción de un indicador de pobreza multidimensional mediante análisis combinatorio
}

\begin{abstract}
Resumen
Se presenta la construcción de modelos de pobreza multidimensional basados en analisis empirico combinatorio y combinatorio. Este enfoque metodologico complementa los modelos tradicionales de pobreza multidimensional, tales como Bourguignon and Chakravarty (2003), Deutch and Silver (2005), Alkire and Foster (2007), Alkire and Foster (2009), entre otros. Estos modelos conservan un análisis unidimensional, y, no desarrollan los atributos entre dimensiones e individuos. Por lo tanto, esta investigación propone complementar y mejorar los modelos existentes anteriormente señalados. Palabras Clave: Pobreza multidimensional, analisis combinatorio, modelos de estimación de pobreza. Clasificación JEL: C02, C65, I32.
\end{abstract}

\section{Combinatory analysis construct a multidimensional poverty indicador}

\begin{abstract}
This document constructs multidimensional poverty models based on the combinatory and mathematic empirical analysis. This methodological approach helps complement traditional multidimensional poverty models, such as: Bourguignon and Chakravarty (2003), Deutch and Silver (2005), Alkire and Foster (2007), Alkire and Foster (2009), among others. These models maintain a unidimensional analysis, even though the relationship protecting dimensions is independent; however, they do not develop the transactional attributes between dimensions and individuals. Therefore, our research is aimed at complementing and improving the existing models above mentioned.
\end{abstract}

Keywords: Multidimensional poverty, combinatory analysis, poverty models conjecture.

JEL Classification: C02, C65, I32.

\section{Construction d'un indicateur multidimensionnel de pauvreté par analyse combinatoire}

\author{
Résumé \\ Ce document utilise l'étude de modèles de pauvreté multidimensionnelle basée sur l'analyse \\ combinatoire empirique et mathématique. Cette méthodologie corrige les méthodes traditionnelles de \\ pauvreté multidimensionnelle (Bourguignon and Chakravarty, 2003; Deutch and Silver, 2005; Alkire \\ and Foster, 2007; Alkire and Foster, 2009). Ces derniers modèles font une analyse unidimensionnelle, \\ ne développant pas les attributs transactionnels entre dimensions et individus. \\ Mots-clés: Pauvreté multidimensionnelle, analyse combinatoire, modèles de conjoncture de pauvreté. \\ Nomenclature JEL: C02, C65, I32.
}




\section{CONSTRUCCIÓN DE UN INDICADOR DE POBREZA MULTIDIMENSIONAL MEDIANTE ANÁLISIS COMBINATORIO}

INFORMACIÓN DEL ARTÍCULO

Recepción de artículo: 2 de marzo de 2016

Aceptación de artículo: 22 de Agosto de 2016
Gary Canaviri-Sillerico Academia Boliviana de Ciencias Económicas Bolivia

Francisco Javier Aliaga-Lordemann Academia Boliviana de Ciencias Económicas

\section{INTRODUCCIÓN}

Una rama importante de las políticas públicas se concentra en la inclusión social y la reducción de la pobreza, esto debido a que más de 1.200 millones de personas viven con 1,25 dólares al día o menos (Naciones Unidas, Objetivos de Desarrollo, 2015). Asimismo, estimaciones del Índice de Pobreza Multidimensional del PNUD (Informe anual del Programa de las Naciones Unidas para el Desarrollo, Tokio 2014) revelan que casi 1.500 millones de personas, de 91 países en desarrollo, viven en situación de pobreza multidimensional, con carencias concurrentes en salud, educación y nivel de vida.

En Bolivia la pobreza monetaria muestra una tendencia decreciente a partir del año 2005, con una tasa de reducción promedio de $4.5 \%$ al año, que representa a 253,364 personas pobres (La evolución de la pobreza en Bolivia: un enfoque multidimensional, Paul Villarroel y Hernani Limarino, 2013). Por otro lado, los resultados sobre pobreza multidimensional muestran que esta se ha reducido en 24 puntos porcentuales durante el periodo 19992011, pero también, se evidencia un aumento de las personas con vulnerabilidad social por el comportamiento elusivo de la pobreza no monetaria en el país.

A pesar de que las medidas presentadas anteriormente permiten examinar la tendencia de la pobreza monetaria y las características de la pobreza multidimensional, existen algunos inconvenientes con su aplicación; la no existencia de una combinación de elementos objetivos ni subjetivos, la falta de herramientas de análisis ante cambios de atributos por dimensión de pobreza, etc.

${ }^{1}$ Autor para correspondencia. Correo electrónico: gaedca_01@icloud.com; javalordeman2@gmail.com 
En consecuencia, es importante construir un modelo que aporte información complementaria.Lainducciónylaconjetura de elementos, permiten la inclusión y el análisis de factores como el trabajo, la salud, la educación, etc., (perspectiva multidimensional). Por otro lado, los cambios de atributos en dimensiones por individuo reflejan permutaciones de datos que se pueden analizar a través del estudio de la combinatoria. Finalmente, la teoría de conjuntos permite la combinación de elementos objetivos y subjetivos del estado de la Pobreza individual y general.

\section{DESARROLLO DE MODELOS DE POBREZA MULTIDIMENSIONAL}

En esta sección se desarrollan modelos de Pobreza multidimensional, en ellos se encotrará la probabilidad del movimiento de atributos por individuo en todas las dimensiones.

Sea $X$ una matriz, con elementos $\chi_{i j} \in X$ donde $\chi_{i j}$ es la cantidad (entero positivo) que la persona $i$ posee del bien o atributo correspondiente a la dimensión $j$. Las filas de la matriz están representadas por los individuos $(i)$, y las columnas por las dimensiones $(j)$ (filas = individuos, columnas = dimensiones). Para cada dimensión se cuenta con una línea de pobreza $\mathrm{Zj}$.

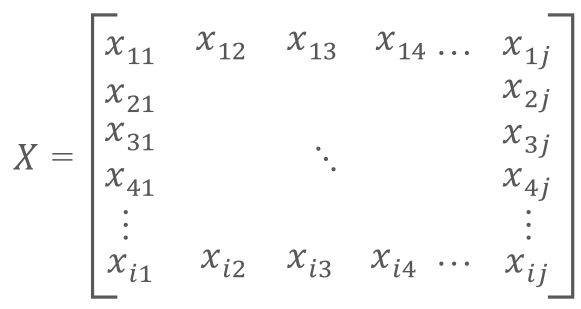

Un individuo $\chi_{i j}$ se considerará pobre en alguna dimensión $j$ si y solo si $\chi_{i j}<Z_{j}$.

$$
A=\left[\begin{array}{ll}
\chi_{11} & \chi_{12}
\end{array}\right]
$$

Un individuo con dos dimensiones, y además suponemos que es pobre simultáneamente en las dos dimensiones, por lo tanto resulta $\chi_{11}<\mathrm{Z}_{1}$ y $\chi_{12}<\mathrm{Z}_{2}$, donde $\mathrm{Z}_{1}$ y $\mathrm{Z}_{2}$ son Líneas de Pobreza de la primera y segunda dimensión respectivamente. El individuo es pobre multidimensional en ambas dimensiones, pero cabe mencionar que solo se midió si es Pobre Multidimensionalmente o no, y no se pudo evaluar la intensidad de los valores que generará una probabilidad mayor o menor de caer en Pobreza Multidimensional, es decir, más allá de encontrar un resultado binario (Pobre ${ }^{-} 1$, No Pobre -0) lo que el documento pretende mostrar es un análisis real de Pobreza, y una tendencia probabilística de caer en Pobreza Multidimensional en todas las dimensiones simultáneamente, para ello es necesario evaluar cada atributo de todas las dimensiones del individuo.

De las cantidades $\chi_{11}$ ó $\chi_{12}$ es posible que disten ciertas unidades ya sea una mayor a otra, en relación a las líneas de pobreza, es decir

$$
\begin{gathered}
\left(\chi_{11} \leq \chi_{12}\right)<\left(Z_{1} \leq Z_{2}\right) \\
\text { ó } \\
\left(\chi_{11} \geq \chi_{12}\right)<\left(Z_{1} \geq Z_{2}\right)
\end{gathered}
$$

Por lo tanto, lo que se medirá es la intensidad de los valores a diferentes cambios de unidades entre esos atributos en relación a las Líneas de Pobreza, en otras palabras la probabilidad de que el individuo incurra en Pobreza Multidimensional en ambas dimensiones simultáneamente, ya sea para matrices $X=\left[\chi_{11}+\chi_{12}+\chi_{13}+\cdots+\chi_{1 n}\right]$ de 2, 3 hasta $n$ dimensiones. 


\section{CONJETURACIÓN PARA UN MODELO DE " $n$ " DIMENSIONES}

Dada la siguiente distribución $\chi_{11}+\chi_{12}+$ $\chi_{13}+\cdots+\chi_{1 n}=w$ y las líneas de Pobreza para cada dimensión $z_{j}$. La probabilidad de incidencia en Pobreza Multidimensional en todas las dimensiones para el individuo $i=1$ está dada por la siguiente expresión.

$$
P_{n}(X, z)=\frac{r}{m}
$$

Donde $r$ es el número de combinaciones posibles que cumplen la condición $\chi_{11}+$ $\chi_{12}+\chi_{13}+\cdots+\chi_{1 n}=w$, donde cada $\chi_{i j}$ se encuentre por debajo de cada línea de pobreza $z_{j} \mathrm{y} m$ será todas las combinaciones de $\chi_{11}+\chi_{12}+\chi_{13}+\cdots+\chi_{1 n}=w$.

Usamos el análisis combinatorio para poder contar las combianciones posibles de $\chi_{11}+\chi_{12}+\chi_{13}+\cdots+\chi_{1 n}$, los patrones de permutación de los elementos de cada fila de la matriz $\mathrm{X}$ siguen un ritmo de (permutaciones con repeticiones), por lo tanto se debe desarrollar las ordenaciones de un número determinado de elementos, se hará un modelo del problema en base a un algoritmo de rayas y asteriscos que nos facilitará encontrar una generalización al mismo.

Se supone el siguiente algoritmo de asteriscos y rayas:

$$
|* * *|
$$

A la izquierda de "| " está la primera columna de la matriz X, entre las "I " está la segunda columna de la matriz $\mathrm{X}, \mathrm{y}$ finalmente al lado derecho de la última " | " está la tercera columna de la matriz. Los asteriscos son elementos iguales, que al contarlos nos dan una cierta cantidad, ej., el algoritmo $|* * *|$ nos refleja que en la primera columna no existe cantidad alguna, en la segunda columna hay tres asteriscos, que es lo mismo a 3 unidades, y en la tercera columna no es existen cantidades.

$$
\text { [ } \left.\begin{array}{lll}
0 & 3 & 0
\end{array}\right]
$$

Veamos otro algoritmo.

$$
*|* * *| *
$$

La relación de la figura nos refleja

$$
\text { [ } \left.\begin{array}{lll}
1 & 3 & 1
\end{array}\right]
$$

Es necesario introducir este sistema ya que la forma de ver las repeticiones tanto en la matriz como en el sistema de asteriscos y rayas llevan la misma estructura de análisis, por lo tanto $\boldsymbol{m}$ será la permutación de $\boldsymbol{w}$ elementos repetidos con $(n-1)$ elementos repetidos, es decir

$$
P_{n}(X, z)=\frac{P R_{w+(n-1)}^{w,(n-1)}-B^{C}}{P R_{w+(n-1)}^{w,(n-1)}}
$$

Donde $r=B=P R_{w+(n-1)}^{w,(n-1)}-B^{C}$, debido a que en la estructura de los conjuntos $\boldsymbol{B}$ y $\boldsymbol{B}^{\boldsymbol{C}}$, el conjunto $\boldsymbol{B}$ tiene los elementos del conjunto solución y que además cumple con las condiciones del problema, es decir $\boldsymbol{r} \in \boldsymbol{B}$, el conunto $\boldsymbol{A}$ es el (conjunto universo) que tiene a todos los elementos permutados, es decir $\boldsymbol{m} \in \boldsymbol{A}$.

$$
B=A-B^{C}
$$

Por lo tanto, se debe encontrar las permutaciones del conjunto $\boldsymbol{B}^{\boldsymbol{C}}$, donde las combinaciones de los elementos $\boldsymbol{B}^{\boldsymbol{C}}$ son aquelllos elementos que se encuentran por encima de la línea de pobreza $\mathrm{Zj}$.

Para hallar el conjunto $\boldsymbol{B}^{C}$ seguimos los siguientes pasos: 


\section{Paso 1}

Analizar el conjunto $\boldsymbol{B}^{C}$ y evaluar los elementos que se encuentren por encima de las líneas de pobreza.

\section{Paso 2}

Llamar delta $(\Delta)$ a las cantidades de igual magnitud que las líneas de pobreza, ya que a partir de ese límite se cumplirá el Paso 1.

$$
\begin{aligned}
& \Delta_{1} \rightarrow \text { primera dimensión } \\
& \Delta_{2} \rightarrow \text { segunda dimensión } \\
& \Delta_{3} \rightarrow \text { tercera dimensión }
\end{aligned}
$$

$$
\Delta_{n} \rightarrow \text { enésima dimensión }
$$

Luego, hallar las combinaciones posibles mayores a cada línea de pobreza

$$
\Delta_{1}|* * * * *| * *|* * * * * * * * * *| * * * *
$$

Para la segunda dimensión

$$
* * * * *\left|\Delta_{2}\right| * *|* * * * * * * * * *| * * * *
$$

Y así sucesivamente, para luego sumar las permutaciones donde cada valor de las líneas de pobreza queden fijas en cada dimensión.

$$
\begin{gathered}
*|* * * *| * *|* *| \ldots \mid \Delta_{n} \\
P R_{\left(w-z_{1}\right)+(n-1)}^{\left(w-z_{1}\right),(n-1)}+P R_{\left(w-z_{2}\right)+(n-1)}^{\left(w-z_{2}\right),(n-1)}+\ldots+P R_{\left(w-z_{n}\right)+(n-1)}^{\left(w-z_{n}\right),(n-1)}
\end{gathered}
$$

Gráfico 1. Intersección de los conjuntos

$$
\Delta_{1}, \Delta_{2}, \Delta_{3}, \Delta_{4}, \Delta_{5}
$$

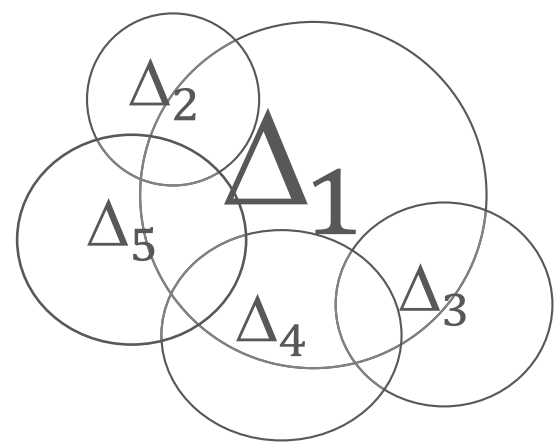

Fuente: Elaboración Propia
Gráfico 2. Intersección de los conjuntos $\Delta_{1}, \Delta_{2} \Delta_{3}$

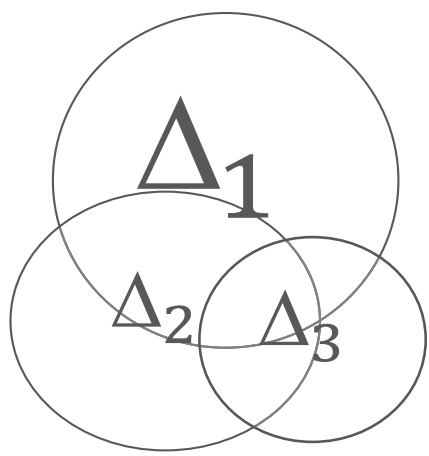

Fuente: Elaboración Propia 


\section{Paso 3}

De hecho, en las permutaciones tomadas anteriormente (paso 2), se analizó los valores por dimensión que se encuentran por encima de los valores de las líneas de pobreza, sería erróneo sumar todos esos valores (paso 2) y restarlos del conjunto $\boldsymbol{A}$ para así llegar a la respuesta, ya que en los valores contados en el paso anterior, existen combinaciones que son contadas varias veces. Reflejamos la situación en el siguiente diagrama, por lo tanto en este paso se tratará de eliminar esas combinaciones.

Eliminando las intersecciones del conjunto de la primera dimensión $\Delta_{1}$ en relación a los otros conjuntos, ejemplo para la intersección de $\Delta_{1}$ y $\Delta_{2}$.

$$
\begin{gathered}
\Delta_{1}\left|\Delta_{2}\right| * *|* * * * * * * * * *| * * * * \\
P R_{\left(w-z_{1}-z_{2}\right)+(n-1)}^{\left(w-z_{1}-z_{2}\right),(n-1)}+P R_{\left(w-z_{1}-z_{3}\right)+(n-1)}^{\left(w-z_{1}-z_{3}\right),(n-1)}+\ldots+P R_{\left(w-z_{1}-z_{n}\right)+(n-1)}^{\left(w-z_{1}-z_{n}\right),(n-1)}
\end{gathered}
$$

Luego, se suman las permutaciones, el cual la primera dimensión es analizada respecto a otra

$$
\Delta_{1}|* *| \Delta_{3}|* *| \ldots \mid
$$

Por otro lado, al descontar la intersección de $\Delta_{2}$ y $\Delta_{3}$, también se reducieron aquellos elementos tomados en la intersección de $\Delta_{1}$ y $\Delta_{2}$. Para ello se debe aumentar la intersección de $\Delta_{1}, \Delta_{2}$ y $\Delta_{3}$

$$
\begin{aligned}
P R_{\left(w-z_{2}-z_{3}\right)+(n-1)}^{\left(w-z_{2}-z_{3}\right),(n-1)}-\left(P R_{\left(w-z_{1}-z_{2}-z_{3}\right)+(n-1)}^{\left(w-z_{1}-z_{2}-z_{3}\right),(n-1)}\right)+P R_{\left(w-z_{2}-z_{4}\right)+(n-1)}^{\left(w-z_{2}-z_{4}\right),(n-1)} & \\
- & \left(P R_{\left(w-z_{1}-z_{2}-z_{4}\right)+(n-1)}^{\left(w-z_{1}-z_{2}-z_{4}\right),(n-1)}\right)+\cdots+P R_{\left(w-z_{2}-z_{n}\right)+(n-1)}^{\left(w-z_{2}-z_{n}\right),(n-1)} \\
- & \left(P R_{\left(w-z_{1}-z_{2}-z_{n}\right)+(n-1)}^{\left(w-z_{1}-z_{2}-z_{n}\right),(n-1)}\right)
\end{aligned}
$$

Asi, sucesivamente y manteniendo la lógica del ejemplo anterior se analizan para la tercera, la cuarta, hasta llegar a la enésima dimensión respecto a las demás.

$$
\begin{aligned}
P R_{\left(w-z_{3}-z_{4}\right)+(n-1)}^{\left(w-z_{3}-z_{4}\right),(n-1)} & \\
& -\left(P R_{\left(w-z_{1}-z_{3}-z_{4}\right)+(n-1)}^{\left(w-z_{1}-z_{3}-z_{4}\right),(n-1)}+P R_{\left(w-z_{2}-z_{3}-z_{4}\right)+(n-1)}^{\left(w-z_{2}-z_{3}-z_{4}\right),(n-1)}\right. \\
& \left.-P R_{\left(w-z_{1}-z_{2}-z_{3}-z_{4}\right)+(n-1)}^{\left(w-z_{1}-z_{2}-z_{3}-z_{4}\right),(n-1)}\right)+\cdots+P R_{\left(w-z_{3}-z_{n}\right)+(n-1)}^{\left(w-z_{3}-z_{n}\right),(n-1)} \\
& -\left(P R_{\left(w-z_{1}-z_{3}-z_{n}\right)+(n-1)}^{\left(w-z_{1}-z_{3}-z_{n}\right),(n-1)}+P R_{\left(w-z_{2}-z_{3}-z_{n}\right)+(n-1)}^{\left(w-z_{2}-z_{3}-z_{n}\right),(n-1)}\right. \\
& \left.-P R_{\left(w-z_{1}-z_{2}-z_{3}-z_{n}\right)+(n-1)}^{\left(w-z_{1}-z_{2}-z_{3}-z_{n}\right),(n-1)}\right)
\end{aligned}
$$




\section{Para Z5}

$P R_{\left(w-z_{4}-z_{5}\right)+(n-1)}^{\left(w-z_{4}-z_{5}\right),(n-1)}$

$$
\begin{aligned}
& -\left(P R_{\left(w-z_{1}-z_{4}-z_{5}\right)+(n-1)}^{\left(w-z_{1}-z_{4}-z_{5}\right),(n-1)}+P R_{\left(w-z_{2}-z_{4}-z_{5}\right)+(n-1)}^{\left(w-z_{2}-z_{4}-z_{5}\right),(n-1)}+P R_{\left(w-z_{3}-z_{4}-z_{5}\right)+(n-1)}^{\left(w-z_{3}-z_{4}-z_{5}\right),(n-1)}\right. \\
& -P R_{\left(w-z_{1}-z_{2}-z_{4}-z_{5}\right)+(n-1)}^{\left(w-z_{1}-z_{2}-z_{4}-z_{5}\right),(n-1)}-P R_{\left(w-z_{1}-z_{3}-z_{4}-z_{5}\right)+(n-1)}^{\left(w-z_{1}-z_{3}-z_{4}-z_{5}\right),(n-1)} \\
& \left.-P R_{\left(w-z_{2}-z_{3}-z_{4}-z_{5}\right)+(n-1)}^{\left(w-z_{2}-z_{3}-z_{4}-z_{5}\right),(n-1)}+P R_{\left(w-z_{1}-z_{2}-z_{3}-z_{4}-z_{5}\right)+(n-1)}^{\left(w-z_{1}-z_{2}-z_{3}-z_{4}-z_{5}\right),(n-1)}\right)+\ldots \\
& +P R_{\left(w-z_{4}-z_{n}\right)+(n-1)}^{\left(w-z_{4}-z_{n}\right),(n-1)} \\
& -\left(P R_{\left(w-z_{1}-z_{4}-z_{n}\right)+(n-1)}^{\left(w-z_{1}-z_{4}-z_{2}\right),(n-1)}+P R_{\left(w-z_{2}-z_{4}-z_{n}\right)+(n-1)}^{\left(w-z_{2}-z_{4}-z_{n}\right),(n-1)}+P R_{\left(w-z_{3}-z_{4}-z_{n}\right)+(n-1)}^{\left(w-z_{3}-z_{4}-z_{n}\right),(n-1)}\right. \\
& -P R_{\left(w-z_{1}-z_{2}-z_{4}-z_{n}\right)+(n-1)}^{\left(w-z_{1}-z_{2}-z_{4}-z_{n}\right),(n-1)}-P R_{\left(w-z_{1}-z_{3}-z_{4}-z_{n}\right)+(n-1)}^{\left(w-z_{1}-z_{3}-z_{4}-z_{n}\right),(n-1)} \\
& \left.-P R_{\left(w-z_{2}-z_{3}-z_{4}-z_{n}\right)+(n-1)}^{\left(w-z_{2}-z_{3}-z_{4}-z_{n}\right),(n-1)}+P R_{\left(w-z_{1}-z_{2}-z_{3}-z_{4}-z_{n}\right)+(n-1)}^{\left(w-z_{1}-z_{2}-z_{3}-z_{4}-z_{n}\right),(n-1)}\right)
\end{aligned}
$$

\section{Para $Z_{6}$}

$$
\begin{aligned}
& P R_{\left(w-z_{5}-z_{6}\right)+(n-1)}^{\left(w-z_{1}-z_{6}\right),(n-1)} \\
& -\left(P R_{\left(w-z_{1}-z_{5}-z_{6}\right)+(n-1)}^{\left(w-z_{1}-z_{5}-z_{6}\right),(n-1)}+P R_{\left(w-z_{2}-z_{5}-z_{6}\right)+(n-1)}^{\left(w-z_{2}-z_{5}-z_{6}\right),(n-1)}+P R_{\left(w-z_{3}-z_{5}-z_{6}\right)+(n-1)}^{\left(w-z_{3}-z_{5}-z_{6}\right),(n-1)}\right. \\
& +P R_{\left(w-z_{4}-z_{5}-z_{6}\right)+(n-1)}^{\left(w-z_{1}-z_{5}-z_{6}\right),(n-1)}-P R_{\left(w-z_{1}-z_{2}-z_{5}-z_{6}\right)+(n-1)}^{\left(w-z_{1}-z_{2}-z_{5}-z_{6}\right),(n-1)} \\
& -P R_{\left(w-z_{1}-z_{3}-z_{5}-z_{6}\right)+(n-1)}^{\left(w-z_{1}-z_{2}-z_{5}-z_{2}\right),(n-1)}-P R_{\left(w-z_{1}-z_{4}-z_{5}-z_{6}\right)+(n-1)}^{\left(w-z_{1}-z_{4}-z_{5}-z_{6}\right),(n-1)} \\
& -P R_{\left(w-z_{2}-z_{3}-z_{5}-z_{6}\right)+(n-1)}^{\left(w-z_{2}-z_{2}-z_{5}-z_{2}\right),(n-1)}-P R_{\left(w-z_{2}-z_{4}-z_{5}-z_{6}\right)+(n-1)}^{\left(w-z_{2}-z_{4}-z_{5}-z_{6}\right),(n-1)} \\
& -P R_{\left(w-z_{3}-z_{4}-z_{5}-z_{6}\right)+(n-1)}^{\left(w-z_{3}-z_{4}-z_{5}-z_{6}\right),(n-1)}+P R_{\left(w-z_{1}-z_{2}-z_{3}-z_{5}-z_{6}\right)+(n-1)}^{\left(w-z_{1}-z_{2}-z_{3}-z_{5}-z_{6}\right),(n-1)} \\
& +P R_{\left(w-z_{1}-z_{2}-z_{4}-z_{5}-z_{6}\right)+(n-1)}^{\left(w-z_{1}-z_{2}-z_{4}-z_{1}-z_{6}\right),(n-1)}+P R_{\left(w-z_{1}-z_{3}-z_{4}-z_{5}-z_{6}\right)+(n-1)}^{\left(w-z_{1}-z_{3}-z_{4}-z_{5}-z_{6}\right),(n-1)} \\
& \left.+P R_{\left(w-z_{2}-z_{3}-z_{4}-z_{5}-z_{6}\right)+(n-1)}^{\left(w-z_{2}-z_{3}-z_{4}-z_{5}-z_{2}\right),(n-1)}-P R_{\left(w-z_{1}-z_{2}-z_{3}-z_{4}-z_{5}-z_{6}\right)+(n-1)}^{\left(w-z_{1}-z_{2}-z_{3}-z_{4}-z_{5}-z_{6}\right),(n-1)}\right)+\ldots \\
& +P R_{\left(w-z_{5}-z_{n}\right)+(n-1)}^{\left(w-z_{5}\right)} \\
& -\left(P R_{\left(w-z_{1}-z_{5}-z_{n}\right)+(n-1)}^{\left(w-z_{1}-z_{5}-z_{n}\right),(n-1)}+P R_{\left(w-z_{2}-z_{5}-z_{n}\right)+(n-1)}^{\left(w-z_{2}-z_{2}-z_{n}\right),(n-1)}+P R_{\left(w-z_{3}-z_{5}-z_{n}\right)+(n-1)}^{\left(w-z_{3}-z_{5}-z_{n}\right),(n-1)}\right. \\
& +P R_{\left(w-z_{4}-z_{5}-z_{n}\right)+(n-1)}^{\left(w-z_{4}-z_{5}-z_{n}\right),(n-1)}-P R_{\left(w-z_{1}-z_{2}-z_{5}-z_{n}\right)+(n-1)}^{\left(w-z_{1}-z_{2}-z_{5}-z_{n}\right),(n-1)} \\
& -P R_{\left(w-z_{1}-z_{3}-z_{5}-z_{n}\right)+(n-1)}^{\left(w-z_{1}-z_{2}-z_{5}-z_{n}\right),(n-1)}-P R_{\left(w-z_{1}-z_{4}-z_{5}-z_{n}\right)+(n-1)}^{\left(w-z_{1}-z_{4}-z_{5}-z_{n}\right),(n-1)} \\
& -P R_{\left(w-z_{2}-z_{3}-z_{5}-z_{n}\right)+(n-1)}^{\left(w-z_{2}-z_{2}-z_{5}-z_{n}\right),(n-1)}-P R_{\left(w-z_{2}-z_{4}-z_{5}-z_{n}\right)+(n-1)}^{\left(w-z_{2}-z_{4}-z_{5}-z_{n}\right),(n-1)} \\
& -P R_{\left(w-z_{3}-z_{4}-z_{5}-z_{n}\right)+(n-1)}^{\left(w-z_{3}-z_{4}-z_{5}-z_{n}\right),(n-1)}+P R_{\left(w-z_{1}-z_{2}-z_{3}-z_{5}-z_{n}\right)+(n-1)}^{\left(w-z_{1}-z_{2}-z_{3}-z_{5}-z_{n}\right),(n-1)} \\
& +P R_{\left(w-z_{1}-z_{2}-z_{4}-z_{5}-z_{n}\right)+(n-1)}^{\left(w-z_{1}-z_{2}-z_{4}-z_{5}\right),(n-1)}+P R_{\left(w-z_{1}-z_{3}-z_{4}-z_{5}-z_{n}\right)+(n-1)}^{\left(w-z_{1}-z_{2}-z_{2}-z_{5}-z_{n}\right),(n-1)} \\
& \left.+P R_{\left(w-z_{2}-z_{3}-z_{4}-z_{5}-z_{n}\right)+(n-1)}^{\left(w-z_{2}-z_{2}-z_{4}-z_{2}-z_{n}\right),(n-1)}-P R_{\left(w-z_{1}-z_{2}-z_{3}-z_{4}-z_{5}-z_{n}\right)+(n-1)}^{\left(w-z_{1}-z_{2}-z_{3}-z_{4}-z_{5}-z_{n}\right),(n-1)}\right)
\end{aligned}
$$




\section{Para $Z_{n}$}

$$
\begin{aligned}
P R_{\left(w-z_{n-1}-z_{n}\right)+(n-1)}^{\left(w-z_{n-1}-z_{n}\right),(n-1)} & \\
& -\left(P R_{\left(w-z_{1}-z_{n-1}-z_{n}\right)+(n-1)}^{\left(w-z_{1}-z_{n-1}-z_{n}\right),(n-1)}+\ldots+P R_{\left(w-z_{n-2}-z_{n-1}-z_{n}\right)+(n-1)}^{\left(w-z_{n-2}-z_{n-1}-z_{n}\right),(n-1)}\right. \\
& -P R_{\left(w-z_{1}-z_{2}-z_{n-1}-z_{n}\right)+(n-1)}^{\left(w-z_{1}-z_{2}-z_{n-1}-z_{n}\right),(n-1)}-\ldots-P R_{\left(w-z_{n-3}-z_{n-2}-z_{n-1}-z_{n}\right)+(n-1)}^{\left(w-z_{n-3}-z_{n-2}-z_{n-1}-z_{n}\right),(n-1)} \\
& +P R_{\left(w-z_{1}-z_{2}-z_{3}-z_{n-1}-z_{n}\right)+(n-1)}^{\left(w-z_{1}-z_{2}-z_{3}-z_{n-1}-z_{n}\right),(n-1)}+\ldots \\
& +P R_{\left(w-z_{n-4}-z_{n-3}-z_{n-2}-z_{n-1}-z_{n}\right)+(n-1)}^{\left(w-z_{n-1}-z_{n-3}-z_{n-2}-z_{n-1}-z_{n}\right),(n-1)}-\ldots \\
& \left.-(-1)^{n} P R_{\left(w-z_{1}-z_{2}-\otimes-z_{n-1}-z_{n}\right)+(n-1)}^{\left(w-z_{1}-z_{2}-\otimes-z_{n-1}-z_{n}\right),(n-1)}\right)
\end{aligned}
$$

Como observamos anteriormente, la relación de números sigue un patrón numérico, y sólo queda sumar los resultados encontrados. Por la cantidad numérica existente tratemos de reducirlos mediante algunos cambios de variable, luego podemos conjeturar la probabilidad y encontrar $\boldsymbol{B}^{C}$.

$$
\begin{aligned}
& B^{C}=\sum_{r=1}^{n} P R_{\left(w-z_{r}\right)+(n-1)}^{\left(w-z_{r}\right),(n-1)} \\
& -\left(\sum_{r=2}^{n} P R_{\left(w-z_{1}-z_{r}\right)+(n-1)}^{\left(w-z_{1}-z_{r}\right)(n-1)}+\sum_{r=3}^{n} P R_{\left(w-z_{2}-z_{r}\right)+(n-1)}^{\left(w-z_{2}-z_{r}\right),(n-1)}+\sum_{r=4}^{n} P R_{\left(w-z_{3}-z_{r}\right)+(n-1)}^{\left(w-z_{3}-z_{r}\right),(n-1)}\right. \\
& +\sum_{r=5}^{n} P R_{\left(w-z_{4}-z_{r}\right)+(n-1)}^{\left(w-z_{4}-z_{r}\right),(n-1)}+\sum_{r=6}^{n} P R_{\left(w-z_{5}-z_{r}\right)+(n-1)}^{\left(w-z_{5}-z_{2}\right),(n-1)} \\
& -\sum_{r=3}^{n} P R_{\left(w-\xi_{1}^{1} z-z_{2}-z_{r}\right)+(n-1)}^{\left(w-\xi_{1}^{1} z-z_{2}-z_{r}\right),(n-1)}-\sum_{r=4}^{n} P R_{\left(w-\xi_{1}^{2} z-z_{3}-z_{r}\right)+(n-1)}^{\left(w-\xi_{1}^{2} z-z_{3}-z_{r}\right),(n-1)} \\
& -\sum_{r=5}^{n} P R_{\left(w-\xi_{1}^{3} z-z_{4}-z_{r}\right)+(n-1)}^{\left(w-\xi_{3}^{3} z-z_{4}-z_{r}\right),(n-1)}-\sum_{r=6}^{n} P R_{\left(w-\xi_{1}^{4} z-z_{5}-z_{r}\right)+(n-1)}^{\left(w-\xi_{1}^{4} z-z_{5}-z_{r}\right),(n-1)} \\
& +\sum_{r=4}^{n} P R_{\left(w-\xi_{2}^{2} z-z_{3}-z_{r}\right)+(n-1)}^{\left(w-\xi_{2}^{2} z-z_{3}-z_{r}\right),(n-1)}+\sum_{r=5}^{n} P R_{\left(w-\xi_{2}^{3} z-z_{4}-z_{r}\right)+(n-1)}^{\left(w-\xi_{3}^{3} z-z_{4}-z_{r}\right),(n-1)} \\
& +\sum_{r=6}^{n} P R_{\left(w-\xi_{2}^{4} z-z_{5}-z_{r}\right)+(n-1)}^{\left(w-\xi_{2}^{4} z-z_{5}-z_{r}\right),(n-1)}-\sum_{r=5}^{n} P R_{\left(w-\xi_{3}^{3} z-z_{4}-z_{r}\right)+(n-1)}^{\left(w-\xi_{3}^{3} z-z_{4}-z_{r}\right),(n-1)} \\
& \left.-\sum_{r=6}^{n} P R_{\left(w-\xi_{3}^{4} z-z_{5}-z_{r}\right)+(n-1)}^{\left(w-\xi_{4}^{4} z-z_{5}-z_{r}\right),(n-1)}+\sum_{r=6}^{n} P R_{\left(w-\xi_{4}^{4} z-z_{5}-z_{r}\right)+(n-1)}^{\left(w-\xi_{4}^{4} z-z_{5}-z_{2}\right),(n-1)}+\ldots\right)
\end{aligned}
$$


En la ecuación anterior introdujimos una variable $\xi$, que refleja la siguiente situación:

$$
\xi_{n}^{m}=\frac{m !}{n ! \cdot(m-n) !}
$$

La relación nos muestra cantidades de combinaciones de $\boldsymbol{m}$ elementos, para formar con ellos $\boldsymbol{n}$ conjuntos, ejemplo.

$$
\begin{aligned}
\sum_{r=4}^{n} P R_{\left(w-\xi_{1}^{2} z-z_{3}-z_{r}\right)+(n-1)}^{\left(w-\xi_{1}^{2} z-z_{3}-z_{r}\right),(n-1)} & \\
& =\left(P R_{\left(w-z_{1}-z_{3}-z_{4}\right)+(n-1)}^{\left(w-z_{1}-z_{3}-z_{4}\right),(n-1)}+P R_{\left(w-z_{2}-z_{3}-z_{4}\right)+(n-1)}^{\left(w-z_{2}-z_{4}\right),(n-1)}\right)+\ldots \\
& +\left(P R_{\left(w-z_{1}-z_{3}-z_{n}\right)+(n-1)}^{\left(w-z_{1}-z_{3}-z_{n}\right),(n-1)}+P R_{\left(w-z_{2}-z_{3}-z_{n}\right),(n-1)}^{\left(w-z_{n}\right)+(n-1)}\right)
\end{aligned}
$$

Es decir $\xi_{n}^{m}$ nos da la cantidad de combinaciones para cada permutación, por lo mostrado continuamos con el desarrollo para obtener $\boldsymbol{B}^{C}$.

$$
\begin{aligned}
B^{C}=\sum_{r=1}^{n} P R_{\left(w-z_{r}\right)+(n-1)}^{\left(w-z_{r}\right),(n-1)} & \left(\sum_{t=1}^{n-1} \sum_{r=t+1}^{n} P R_{\left(w-z_{t}-z_{r}\right)+(n-1)}^{\left(w-z_{t}-z_{r}\right),(n-1)}-\sum_{t=2}^{n-1} \sum_{r=t+1}^{n} P R_{\left(w-\xi_{1}^{t-1} z-z_{t}-z_{r}\right)+(n-1)}^{\left(w-\xi_{1}^{t-1} z-z_{t}-z_{r}\right),(n-1)}\right. \\
& +\sum_{t=3}^{n-1} \sum_{r=t+1}^{n} P R_{\left(w-\xi_{2}^{t-1} z-z_{t}-z_{r}\right)+(n-1)}^{\left(w-\xi_{t-1}^{t-1} z-z_{t}-z_{r}\right),(n-1)}-\sum_{t=4}^{n-1} \sum_{r=t+1}^{n} P R_{\left(w-\xi_{3}^{t-1} z-z_{t}-z_{r}\right)+(n-1)}^{\left(w-\xi^{t-1} z-z_{t}-z_{r}\right),(n-1)} \\
& \left.+\sum_{t=5}^{n-1} \sum_{r=t+1}^{n} P R_{\left(w-\xi_{4}^{t-1} z-z_{t}-z_{r}\right)+(n-1)}^{\left(w-\xi^{t-1} z-z_{t}-z_{r}\right),(n-1)}-\ldots\right)
\end{aligned}
$$

Ordenando algebraicamente, llegamos al resultado.

$$
B^{C}=\sum_{r=1}^{n} P R_{\left(w-z_{r}\right)+(n-1)}^{\left(w-z_{r}\right),(n-1)}-\sum_{s=0}^{n-2}(-1)^{s} \sum_{t=s+1}^{n-1} \sum_{r=t+1}^{n} P R_{\left(w-\xi_{s}^{t-1} z-z_{t}-z_{r}\right)+(n-1)}^{\left(w-\xi_{s}^{t-1} z-z_{t}-z_{r}\right),(n-1)}
$$

Reemplazamos el resultado obtenido en la ecuación $\boldsymbol{B}-\boldsymbol{A}-\boldsymbol{B}^{\boldsymbol{C}}$

$$
\begin{aligned}
B=A-B^{C}= & P R_{w+(n-1)}^{w,(n-1)} \\
& -\left(\sum_{r=1}^{n} P R_{\left(w-z_{r}\right)+(n-1)}^{\left(w-z_{r}\right),(n-1)}-\sum_{s=0}^{n-2}(-1)^{s} \sum_{t=s+1}^{n-1} \sum_{r=t+1}^{n} P R_{\left(w-\xi_{s}^{t-1} z-z_{t}-z_{r}\right)+(n-1)}^{\left(w-\xi_{s}^{t-1} z-z_{t}-z_{r}\right),(n-1)}\right)
\end{aligned}
$$


Por lo tanto, la probabilidad que tiene un individuo para estar en Pobreza Multidimensional en todas las dimensiones seleccionadas, según las combinaciones de los atributos por cada dimensión, dadas diferentes líneas de pobreza y una extensión hasta $\boldsymbol{n}$ dimensiones es:

$$
\begin{aligned}
P R_{w+(n-1)}^{w,(n-1)}[1- & \left.P_{n}(X, z)\right] \\
& =\left(\sum_{r=1}^{n} P R_{\left(w-z_{r}\right)+(n-1)}^{\left(w-z_{r}\right),(n-1)}-\sum_{s=0}^{n-2}(-1)^{s} \sum_{t=s+1}^{n-1} \sum_{r=t+1}^{n} P R_{\left(w-\xi_{s}^{t-1} z-z_{t}-z_{r}\right)+(n-1)}^{\left(w-\xi_{s}^{t-1} z-z_{t}-z_{r}\right),(n-1)}\right)
\end{aligned}
$$

\section{COROLARIOS}

Por lo expuesto anteriormente la ecuación

$$
P_{n}(X, z)=\frac{A-B^{C}}{A}
$$

Encuentra la probabilidad de que un individuo se encuentre en Pobreza Multidimensional en todas las dimensiones seleccionadas dado el número de posibles combinaciones que se pueden obtener permutando los atributos de todas las dimensiones.

El conjunto $\frac{A-B^{C}}{A}$ refleja tal situación, ahora tomemos el complemento de este conjunto,

$$
1-\frac{A-B^{C}}{A}
$$

Ordenando algebraicamente

$$
\begin{gathered}
P^{n}(X, z)=1-P_{n}(X, z)=\frac{B^{C}}{A} \\
P^{n}(X, z)=\frac{B^{C}}{A}
\end{gathered}
$$

Reemplazando

$P^{n}(X, z)=\frac{\sum_{r=1}^{n} P R_{\left(w-z_{r}\right)+(n-1)}^{\left(w-z_{r}\right),(n-1)}-\sum_{s=0}^{n-2}(-1)^{s} \sum_{t=s+1}^{n-1} \sum_{r=t+1}^{n} P R_{\left(w-\xi_{s}^{t-1} z-z_{t}-z_{r}\right)+(n-1)}^{\left(w-\xi_{t}^{t}{ }^{1} z_{t}-z_{r}\right),(n-1)}}{P R_{w+(n-1)}^{w,(n-1)}}$

Esta probabilidad nos muestra la situación de un individuo en no estar en Pobreza Multidimensional en todas las dimensiones seleccionadas, dado el número de posibles combinaciones que se pueden obtener permutando los atributos de todas las dimensiones. 


\section{CONJETURACIÓN PARA UN MODELO DE "n" INDIVIDUOS}

Veremos un corolario al primer modelo, la diferencia estará en que se tomará sólo una Línea de Pobreza ya que el análisis se hará por dimensión. En el modelo anterior vimos un desarrollo de la Pobreza Multidimensional tomando como una variable dependiente al individuo y analizando bajo ese punto de vista las dimensiones, ahora en esta segunda parte analizaremos inversamente, es decir tomaremos a la dimensión como una variable dependiente y a los individuos como variables independientes.

$P^{m}(X, Z)$
$=\frac{P R_{n+(m-1)}^{n,(m-1)}-\left(P R_{m}^{1,(m-1)} \cdot P R_{\left(n-z_{j}\right)+(m-1)}^{\left(n-z_{j}\right),(m-1)}-\left(P R_{m}^{2,(m-2)} \cdot P R_{\left(n-2 z_{j}\right)+(m-1)}^{(n-2 z)(m-1)}-\ldots-\left(P R_{m}^{(m-2), 2} \cdot P R_{\left(n-(m-2) z_{j}\right)+(m-1)}^{\left(n-(m-2) z_{j}(m-1)\right.}-P R_{m}^{(m-1), 1} \cdot P R_{\left(n-(m-1) z_{j}\right)+(n-1)}^{\left(n-(m-1) z_{j}(n-1)\right.}\right)\right)\right)}{P R_{n+(m-1)}^{n,(m-1)}}$

Para encontrar la probabilidad de no pobreza en cada dimensión, bastará restar el complemento al caso general de $\boldsymbol{n}$ individuos.

Para todo $\mathrm{z}_{j}$ con $\boldsymbol{i}=\boldsymbol{m}$ individuos y con los atributos $\sum_{i=1}^{n} x_{i j}$, la probabilidad de no pobreza de cada dimensión en cada individuo está dada por

$$
P^{m}(X, z)=\frac{P R_{\left(n-m \cdot z_{j}\right)+(m-1)}^{\left(n-m \cdot z_{j}\right),(m-1)}}{P R_{n+(m-1)}^{n,(m-1)}}
$$

\section{CONCLUSIONES}

A lo largo de la presente investigación se demostró la posibilidad de seccionar la población y las dimensiones de la pobreza multidimensional. Este resultado permite mejorar el problema cualitativo presente en Bourguignon y Chakravarty (2003), quienes no analizan la sensibilidad de la entrada y salida de la pobreza en función de cambios en atributos entre individuos y dimensiones.

Por otro lado, se extiende el modelo presentado por Chakravarty, Deutsch y Silver (2005) y Alkire y Foster (2007), quienes consideran la relación entre las dimensiones como factores independientes. El modelo propuesto en este trabajo, asume una relación de dependencia entre las dimensiones (ya que se analiza la suma total de éstas). De esta forma, la dependencia permite que un cambio en las unidades de los atributos afecte el resultado general.

Al igual que en el modelo de Alkire y Foster (2009, CONEVAL) los modelos desarrollados permiten distinguir la población pobre y vulnerable, y adicionalmente resuelven el problema de la sustitución de dimensiones. La idea de la dinámica de la pobreza (Decomposing Multidimensional Poverty Dynamics de Mauricio Apablaza y GastonYalonetzky, 2013) se complementó ya que se estudiaron las transiciones de cambios de atributos entre dimensiones e individuos.

Finalmente, los modelos desarrollados fueron analizados bajo la óptica de la lógica difusa, pues no poseen un valor 
máximo entre los datos de los atributos en dimensiones e individuos. Estudios posteriores que utilicen la teoría de conjuntosyla lógica difusa parala medición de la pobreza multidimensional, deberán analizar pronósticos de índices definidos entre 0 e infinito, para caracterizar el modo social de organización de la pobreza.

\section{REFERENCIAS BIBLIOGRÁFICAS}

Alarcón. (2001). Medición de las condiciones de vida. Instituto Interamericano para el Desarrollo Social, INDES (págs. 1-21). Banco Interamericano de Desarrollo, Series documentos de trabajo.

Alkire, \& Foster. (2007). Counting and Multidimensional Poverty Measurement. Working Paper Series, OPHI.

Alkire, S. (2010). Iniciativa de Desarrollo Humano y reducción de la pobreza. Obtenido de Departamento de Desarrollo Internacional, Universidad de Oxford: http://www.ophi.org.ok

Apablaza, M., \& Yalonetsky, G. (2013). Descomposing Multidimensional Poverty Dinamics. Reino Unido.

Badenés Plá, N. (2014). El modelo de Rash aplicado a la medición multidimensional de la pobreza. España.

Banco Mundial. (2000). Informe sobre el desarrollo mundial 2000/2001: Lucha contra la pobreza. Washington DC. Oxford University Press.

Becker, M., Pietrocola, N., \& Sanchez, C. (1996). Notas de Combinatoria. Buenos Aires: Red Olímpica.

Berenger, V., Calderón Villarreal, C., \& Celestine, F. (2009). Modelling the Distribution of Multidimensional Poverty Scores. México.

Bourguignon, \& Chakravarty. (2003). The measurement of multidimensional poverty. Journal of Economic Inequality 1.
CEPAL. (2010). Guía para estimar la pobreza infantil.

Conconi, \& Ham. (2008). Pobreza Multidimensional, una aplicación a la Argentina. Argentina: Working Papers 0057, CEDLAS.

Conconi, A. (2004). Pobreza Multidimensional en Argentina. Argentina.

CONEVAL-MÉXICO. (2009). Generación de un índice multidimensional de pobreza. México: Documentos de trabajo.

Denis, A., Gallegos, F., \& Sanhuesa, C. (2010). Medición de la Pobreza Multidimensional en Chile. Chile: Universidad Alberto Hurtado.

Eid, A., \& Aliaga Lordemann, J. (2013). "Vivir Bien" y pobreza multidimensional ¿Es posible una conciliación conceptual? Revista Latinoamericana de Desarrollo Económico, 231-253.

Feller. (2010). An introduction to Probability Theory Vol.1. Pensilvania: Carnegie Mellon University.

Feres, \& Mancero. (2001). Enfoques para la medición de la pobreza. Series Estudios Estadísticos y Prospectivos No 4 CEPAL.

Fonseca Godinez, C. E. (2010). El enfoque de conjuntos difusos para la medición multidimensional de la pobreza. México.

Foster, Greer, \& Thorbecke. (1984). A class of decomposable poverty measures, Econométrica. Vol. 52.

Galindo Gómez. (2014). Conjuntos y sistemas difusos. España.

Grinstead, \& Snell. (2004). Cadenas de Markov. Colorado: CSM.

Grupo de Río. (2007). Compendio de mejores prácticas en la medición de la pobreza. Santiago de Chile: Capítulo 2.

Hurtado, A. (2010). A new technique to measure poverty using the theory of fuzzy logic. OXFORD. 
López Calva, L., \& Rodriguez, L. (2005). Muchos rostros, un sólo espejo: Restricciones para la medición multidimensional de la pobreza en México. Série documentos de investigación 20, SEDESOL.

México, I. N. (2010). Estadísticas a propósito del día mundial de la justicia social. México.

Nieto Said, J. (2014). Libro de Combinatoria. Argentina.

Pérez Segui, M. L. (2003). Combinatoria. Distrito Federal: Cuaderno de Olimpiadas Matemáticas, UNAM, México.

Perticara, \& Sanhueza. (2008). La compleja tarea de identificar quien es pobre. Chile: Observatorio Económico, Universidad Alberto Hurtado.

PNUD. (2010). Human Progress and the rising shout.
Pugachev. (2001). Introducción a la teoría de las Probabilidades. California: UCLA.

Reyes, C. (2012). Apuntes de Inducción Matemática. México.

Rojas Aguilar, J. L. (2014). Modelando la Pobreza Multidimensional, un análisis estocástico. México.

Villarroel, P., \& Hernani Limarino, W. (2013). La evolución de la pobreza en Bolivia: un enfoque multidimensional. Revista Latinoamericana de Desarrollo Económico, 7-72.

Wilhelmi, M. (2005). Combinatoria y Probabilidad. Granada: Universidad de Granada, España.

Canaviri G., \& Aliaga-Lordemánn, J. (2016). Combinatory analysis construct a multidimensional poverty indicador. Panorama Económico, 24, 85-104.

\section{AUTORES}

Gary Canaviri-Sillerico

Licenciado en Ciencias Económicas de la Universidad Católica Boliviana (Bolivia), Licenciado en Matemática de la Universidad Mayor de San Andrés (Bolivia), Investigador Independiente. Analista en Pobreza Multidimensional. Actualmente Miembro de la Sociedad Boliviana de Ciencias, y miembro de Kangouru sans Frontieres (Bolivia).

Francisco Javier Aliaga-Lordemann

Doctor y Magister en economía en la Universidad de Zaragoza (España). Desde 2010 es miembro de número de la Academia Boliviana de Ciencias Económicas, en 2011 recibió el Premio Bernard Prince de Investigación en Holanda. Se ha desempeñado como Director de Investigación, académico, consultor internacional, asesor económico-financiero en más de 25 países para diversas instituciones como el BID, BM, GIZ, UE, FMI, IDRC,MCC, UN, FAO,CAF, Bank of Asia, Bank of África, etc. Realizando misiones en Centro América, Europa. Asia y África, en temas de economía de la infraestructura, economía experimental, cambio climático, pobreza, energías renovables y economía de la felicidad. 


\section{ANEXOS}

\section{Combinatoria}

Una de las nociones matemáticas abstractas mas remotas es la del número, y dentro de ellos el de los números naturales o enteros positivos: $1,2,3,4,5$, etc. Con ello representamos las cantidades de objetos que se presentan en la vida cotidiana, y la combinatoria nos facilita ilustrar la necesidad de aprender estas técnicas de conteo (Pérez Segui, 2003). La combinatoria es la abstracción de un problema reducido en una fórmula con una operación básica de la aritmética como es la multiplicación (Becker, Pietrocola, \& Sanchez, 1996). En el desarrollo del modelo una de las técnicas que se usa es la del conteo con repetición, a continuación su definición ${ }^{1}$.

\section{i. Definición}

Dada una colección de $n$ objetos $\boldsymbol{a}_{1}, \boldsymbol{a}_{2}, \ldots$ $\boldsymbol{a}_{\mathrm{n}}$, llamaremos permutación a cualquier ordenación de los mismos. Por tanto, dos permutaciones serán distintas si los objetos están colocados en orden diferente.

Por ejemplo, en una colección de cinco objetos, $\boldsymbol{a}_{1}, \boldsymbol{a}_{2}, \boldsymbol{a}_{3}, \boldsymbol{a}_{4}$, y $\boldsymbol{a}_{5}$ dos permutaciones distintas de ellos serán:

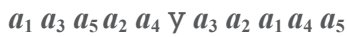

\section{ii. Número de Permutaciones}

El número de permutaciones de $\boldsymbol{n}$ objetos lo designamos por $\boldsymbol{P}_{\boldsymbol{n}}$ y su valor por el principio de multiplicación es

$$
P_{n}=1 \cdot 2 \cdot 3 \cdot 4 \cdot \ldots \ldots \cdot(n-1) \cdot n
$$

1 Notas de Combinatoria, Becker pág. 7 (red Olímpica, Argentina 1996)
A este número se le llama factorial de $\boldsymbol{n}$ y se anota $n$ !, es decir,

$$
n !=n \cdot(n-1) \cdot \ldots \ldots \cdot 3 \cdot 2 \cdot 1
$$

Obsérvese que

$$
n !=n \cdot(n-1) !
$$

iii. Permutaciones con repetición

Sea una colección de $\boldsymbol{n}$ objetos entre los que hay $\boldsymbol{n}_{\mathbf{1}}$ iguales entre sí, $\boldsymbol{n}_{\mathbf{2}}$ iguales entre sí pero distintos de los $\boldsymbol{n}_{\mathbf{1}}, \boldsymbol{n}_{\mathbf{3}}$ iguales entre sí, pero distintos de $\operatorname{los} \boldsymbol{n}_{1}$ y $\boldsymbol{n}_{2}$ y así sucesivamente hasta $\boldsymbol{n}_{r}$ iguales entre sí, pero distintos de todos los anteriores (Wilhelmi, 2005). Llamaremos permutaciones con repetición a las distintas formas de ordenarlos.

Obsérvese que dos permutaciones cualesquiera serán diferentes, cuando se diferencian, al menos, por el lugar que ocupan dos elementos distintos.

Las denotaremos por

$$
P R_{n}^{n_{1}, n_{2}, \ldots, n_{r}}
$$

donde, $\boldsymbol{n}_{1}+\boldsymbol{n}_{2}+\boldsymbol{n}_{3}+\cdots+\boldsymbol{n}_{r}=\boldsymbol{n}$

iv. Número de permutaciones con repetición ${ }^{2}$

El número de permutaciones con repetición de $\boldsymbol{n}$ elementos en las condiciones de la definición anterior es

$$
P R_{n}^{n_{1}, n_{2}, \ldots, n_{r}}=\frac{P_{n}}{P_{n_{1}} \cdot P_{n_{2}} \cdot \ldots \cdot P_{n_{r}}}=\frac{n !}{n_{1} ! \cdot n_{2} ! \cdot \ldots \cdot n_{r} !}
$$

2 Combinatoria, María Luisa Pérez pág. 75 (UNAM, 2004) 


\section{Ejemplo numérico, caso $\boldsymbol{j}=\mathbf{3}$ (tres dimensiones).}

A continuación se muestra el desarrollo para el análisis con 3 dimensiones, para tal efecto usaremos la combinatoria ya que permutar números con tres dimensiones nos obliga a abstraer el problema, veamos un ejemplo cuando $\left(\chi_{11}+\chi_{12}+\chi_{13}=11\right)$.
Dado un individuo y la condición $\chi_{11}$ $+\chi_{12}+\chi_{13}=11$, con Líneas de Pobreza $\mathrm{Z}_{1}=5, \mathrm{Z}_{2}=3, \mathrm{Z}_{3}=6$, en las dimensiones 1,2 y 3 respectivamente. Se forman las combinaciones de números que son $\chi_{11}$, $\chi_{12}, \chi_{13}$ enteros positivos y que además cumplen la condición $\chi_{11}+\chi_{12}+\chi_{13}=11$.

Tabla 1. Intercambio de atributos para $\left(X_{11}+X_{12}+X_{13}=11\right)$.

\begin{tabular}{|c|c|c|}
\hline 0 & 0 & 11 \\
\hline 0 & 1 & 10 \\
\hline 0 & 2 & 9 \\
\hline 0 & 3 & 8 \\
\hline 0 & 4 & 7 \\
\hline 0 & 5 & 6 \\
\hline 0 & 6 & 5 \\
\hline 0 & 7 & 4 \\
\hline 0 & 8 & 3 \\
\hline 0 & 9 & 2 \\
\hline 0 & 10 & 1 \\
\hline 0 & 11 & 0 \\
\hline 1 & 0 & 10 \\
\hline 1 & 1 & 9 \\
\hline 1 & 2 & 8 \\
\hline
\end{tabular}

\begin{tabular}{|c|c|c|}
\hline 1 & 3 & 7 \\
\hline 1 & 4 & 6 \\
\hline 1 & 5 & 5 \\
\hline 1 & 6 & 4 \\
\hline 1 & 7 & 3 \\
\hline 1 & 8 & 2 \\
\hline 1 & 9 & 1 \\
\hline 1 & 10 & 0 \\
\hline 2 & 0 & 9 \\
\hline 2 & 1 & 8 \\
\hline 2 & 2 & 7 \\
\hline 2 & 3 & 6 \\
\hline 2 & 4 & 5 \\
\hline 2 & 5 & 4 \\
\hline 2 & 6 & 3 \\
\hline
\end{tabular}

\begin{tabular}{|l|l|l|}
\hline 2 & 7 & 2 \\
\hline 2 & 8 & 1 \\
\hline 2 & 9 & 0 \\
\hline 3 & 0 & 8 \\
\hline 3 & 1 & 7 \\
\hline 3 & 2 & 6 \\
\hline 3 & 3 & 5 \\
\hline 3 & 4 & 4 \\
\hline 3 & 5 & 3 \\
\hline 3 & 6 & 2 \\
\hline 3 & 7 & 1 \\
\hline 3 & 8 & 0 \\
\hline 4 & 0 & 7 \\
\hline 4 & 1 & 6 \\
\hline 4 & 2 & 5 \\
\hline 4 & 3 & 4 \\
\hline
\end{tabular}

\begin{tabular}{|l|l|l|}
\hline 4 & 4 & 3 \\
\hline 4 & 5 & 2 \\
\hline 4 & 6 & 1 \\
\hline 4 & 7 & 0 \\
\hline 5 & 0 & 6 \\
\hline 5 & 1 & 5 \\
\hline 5 & 2 & 4 \\
\hline 5 & 3 & 3 \\
\hline 5 & 4 & 2 \\
\hline 5 & 5 & 1 \\
\hline 5 & 6 & 0 \\
\hline 6 & 0 & 5 \\
\hline 6 & 1 & 4 \\
\hline 6 & 2 & 3 \\
\hline 6 & 3 & 2 \\
\hline 6 & 4 & 1 \\
\hline
\end{tabular}

\begin{tabular}{|c|c|c|}
\hline 6 & 5 & 0 \\
\hline 7 & 0 & 4 \\
\hline 7 & 1 & 3 \\
\hline 7 & 2 & 2 \\
\hline 7 & 3 & 1 \\
\hline 7 & 4 & 0 \\
\hline 8 & 0 & 3 \\
\hline 8 & 1 & 2 \\
\hline 8 & 2 & 1 \\
\hline 8 & 3 & 0 \\
\hline 9 & 0 & 2 \\
\hline 9 & 1 & 1 \\
\hline 9 & 2 & 0 \\
\hline 10 & 0 & 1 \\
\hline 10 & 1 & 0 \\
\hline 11 & 0 & 0 \\
\hline
\end{tabular}

Fuente: Elaboración Propia

La probabilidad que el individuo se encuentre en Pobreza Multidimensional en las tres dimensiones es

$$
P_{3}(X, z)=\frac{r}{m}
$$

Donde $\boldsymbol{r}$ será el número de combinaciones posibles que cumplan la condición $\chi_{11}+$ $\chi_{12}+\chi_{13}=11$ y que estén por debajo de las líneas de pobreza $\mathrm{Z}_{1}=5, \mathrm{Z}_{2}=3$ y $\mathrm{Z}_{3}=6, \boldsymbol{m}$ será todas las combinaciones de $\chi_{11}+\chi_{12}+$ $\chi_{13}=11$.
En nuestro ejemplo

$$
\chi_{11}+\chi_{12}+\chi_{13}=11
$$

Algunas combinaciones serían

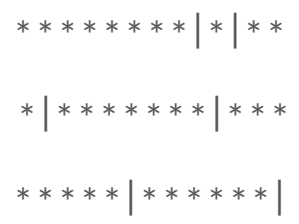


Que generan

$$
\text { [812] [173] [560] }
$$

\section{Respectivamente}

De esta forma es posible generar muchas combinaciones, por lo tanto para encontrar el valor de "m" solo bastará hallar el número de permutaciones posibles del sistema de 11 asteriscos y 2 rayas que representa la suma de $\chi_{11}+\chi_{12}+\chi_{13}=11$. Para ello se usa el teorema de Número de Permutaciones con repetición.

Por lo tanto, el Número de Permutaciones con repetición del sistema

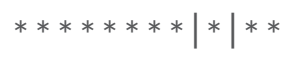

es

$$
P R_{13}^{11,2}=\frac{13 !}{11 ! \cdot 2 !}
$$

Por el teorema del número de permutaciones tenemos

$$
13 !=13 \cdot 12 \cdot 11 !
$$

Reemplazando

$P R_{13}^{11,2}=\frac{13 !}{11 ! \cdot 2 !}=\frac{13 \cdot 12 \cdot 11 !}{11 ! \cdot 2 \cdot 1}=\frac{13 \cdot 12}{2 \cdot 1}=78$

Por lo tanto $\boldsymbol{m}=78$

Para hallar el valor de $r$ será necesario entrar a un análisis aún más complejo. Según la grilla de números mostrados anteriormente y que representan todas las permutaciones de $\chi_{11}+\chi_{12}+\chi_{13}=$ 11 se deduce que $r=1\left(\begin{array}{lll}4 & 2 & 5\end{array}\right)$ visto anteriormente, $\mathrm{y}$ es la única posibilidad de permutación que cumple $\chi_{11}+\chi_{12}+\chi_{13}=$ 11 y está por debajo de $\mathrm{Z}_{1}=5, \mathrm{Z}_{2}=3 \mathrm{y} \mathrm{Z}$ $=6$, para otros problemas sería una tarea muy complicada si realizamos todas las combinaciones posibles y buscamos cuál de ellas es solución a nuestro sistema, para ello se requiere una forma que nos brinde el modo más adecuado y fácil de encontrar el conjunto de posibles soluciones.

Sea el siguiente sistema de conjuntos

$$
A=B \cup B^{C}
$$

Donde $A$ es el total de permutaciones posibles del sistema de asteriscos y rayas, que en todo caso es el valor encontrado $\boldsymbol{m}$ $=78, \boldsymbol{B}$ es el conjunto de permutaciones posibles que están por debajo de $\mathrm{Z}_{1}=$ $5, Z_{2}=3$ y $Z_{3}=6$, y $B^{C}$ es el conjunto de permutaciones posibles que están por encima de $\mathrm{Z}_{1}=5, \mathrm{Z}_{2}=3 \mathrm{y} \mathrm{Z}_{3}=6$.

Por lo tanto en el conjunto $B$ se muestra una posible situación.

Tabla 2: Posible situación para $\mathrm{z}_{1}<5, \mathrm{z}_{2}<3$ y $\mathrm{z}_{3}<6$.

\begin{tabular}{|c|c|c|}
\hline$<5$ & $<3$ & $<6$ \\
\hline$* * * *$ & $* *$ & $* * * * *$ \\
\hline
\end{tabular}

Fuente: Elaboración Propia

Se deben permutar los asteriscos con la condición de no ser mayor ni igual a 5, 3 y 6 en la primera, segunda y tercera casilla respectivamente, y además deben cumplir con esas desigualdades simultáneamente.

El conjunto $\boldsymbol{B}^{C}$ tendrá una posible situación

Tabla 3: Posible situación para $\mathrm{z}_{1} \geq 5, \mathrm{z}_{2} \geq 3$ y $\mathrm{z}_{3} \geq 6$.

\begin{tabular}{|c|c|c|}
\hline$\geq 5$ & $\geq 3$ & $\geq 6$ \\
\hline$* * * * *$ & $* * *$ & $* * * * * *$ \\
\hline
\end{tabular}

Fuente: Elaboración Propia

Donde deben permutar los asteriscos con la siguiente condición; en cada casilla se debe tener una cantidad mayor o igual a 
5,3 y 6 en la primera, segunda y tercera casilla respectivamente, y que a lo sumo cumpla con una de las tres condiciones, aquí no es necesario que se cumplan las desigualdades simultáneamente, por el hecho que este conjunto es el complemento del anterior.

Si vemos con detalle la estructura de los conjuntos $\boldsymbol{B}$ у $\boldsymbol{B}^{\boldsymbol{C}}$, el conjunto $\boldsymbol{B}$ tiene los elementos del conjunto solución y que además cumple con las condiciones del problema, es decir $\boldsymbol{r} \in \boldsymbol{B}$, el cojunto A es el (conjunto universo) que tiene a todos los elementos permutados, es decir $\boldsymbol{m} \in \boldsymbol{A}$.

$$
B-A-B^{C}
$$

Por lo tanto, se debe encontrar las permutaciones del conjunto $\boldsymbol{B}^{C}$, es decir el análisis se enfoca en encontrar permutaciones de asteriscos que tengan a lo mínimo cantidades de 5, 3 y 6 en cada casilla respectivamente.

Para empezar se analiza la primera casilla, y se realiza el conteo de las soluciones en las que se obtengan cantidades de asteriscos iguales o mayores a 5 .

Se presenta una posible solución

$$
* * * * *|* * *| * * *
$$

Para comprender el sistema se crea un conjunto $\Delta$ (delta) que contenga 5 asteriscos como elementos

$$
\Delta=* * * * *
$$

El sistema anterior se refleja como

$$
\Delta=|* * *| * * *
$$

Por lo tanto si dejamos $\Delta$ (delta) en la primera casilla y permutan los demás elementos, tendremos un sistema de 8 elementos con 6 asteriscos y 2 rayas.

$$
P R_{8}^{6,2}=\frac{8 !}{6 ! \cdot 2 !}=28
$$

Se toman en cuenta las siguientes cantidades de números.

Tabla 4: Intercambio de atributos para

$\left(X_{11}+X_{12}+X_{13}=11\right)$, cuando $X_{11} \geq 5$

\begin{tabular}{|l|l|l|}
\hline 5 & 0 & 6 \\
\hline 5 & 1 & 5 \\
\hline 5 & 2 & 4 \\
\hline 5 & 3 & 3 \\
\hline 5 & 4 & 2 \\
\hline 5 & 5 & 1 \\
\hline 5 & 6 & 0 \\
\hline 6 & 0 & 5 \\
\hline 6 & 1 & 4 \\
\hline 6 & 2 & 3 \\
\hline 6 & 3 & 2 \\
\hline 6 & 4 & 1 \\
\hline 6 & 5 & 0 \\
\hline
\end{tabular}

\begin{tabular}{|c|c|c|}
\hline 7 & 0 & 4 \\
\hline 7 & 1 & 3 \\
\hline 7 & 2 & 2 \\
\hline 7 & 3 & 1 \\
\hline 7 & 4 & 0 \\
\hline 8 & 0 & 3 \\
\hline 8 & 1 & 2 \\
\hline 8 & 2 & 1 \\
\hline 8 & 3 & 0 \\
\hline 9 & 0 & 2 \\
\hline 9 & 1 & 1 \\
\hline 9 & 2 & 0 \\
\hline 10 & 0 & 1 \\
\hline 10 & 1 & 0 \\
\hline 11 & 0 & 0 \\
\hline
\end{tabular}

Fuente: Elaboración Propia

Ahora, se analiza de la misma forma con la segunda casilla, es decir

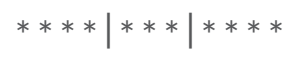

$\Delta_{1}$ (delta sub uno) representa a los 3 asteriscos de la segunda casilla

$$
\Delta_{1}=* * *
$$

por lo que tendremos 


$$
* * * *\left|\Delta_{1}\right| * * * *
$$

Si mantenemos $\Delta_{1}$ en la segunda casilla y permutamos los demás elementos, tendremos un sistema de 10 elementos con 8 asteriscos y 2 rayas.

$$
P R_{10}^{8,2}=\frac{10 !}{8 ! \cdot 2 !}=45
$$

Análogamente ocurrirá con la tercera casilla, donde $\Delta_{2}$ representa a 6 asteriscos,

$$
\begin{gathered}
* * *|* *| * * * * * * \\
\triangle_{2}=* * * * * * \\
* * *|* *| \triangle_{2}
\end{gathered}
$$

Por lo tanto dejemos $\Delta_{2}$ en la tercera casilla y permutemos los demas elementos, lo cual tendremos un sistema de 7 elementos con 5 asteriscos y 2 rayas.

$$
P R_{7}^{5,2}=\frac{7 !}{5 ! \cdot 2 !}=21
$$

Ahora, al unir los tres conjuntos $\Delta \cup \Delta_{1}$ $\cup \Delta_{2}$, éstos generan elementos repetidos como son:

Tabla 5: Elementos repetidos en el intercambio de atributos para $\left(X_{11}+X_{12}+X_{13}=11\right)$, cuando $X_{11} \geq 5, X_{12} \geq 3$

\begin{tabular}{|c|c|c|}
\hline 5 & 3 & 3 \\
\hline 5 & 4 & 2 \\
\hline 5 & 5 & 1 \\
\hline 5 & 6 & 0 \\
\hline
\end{tabular}

Fuente: Elaboración Propia

Que son elementos tanto de los conjuntos $\Delta \mathrm{y} \Delta_{1}$. Ocurre lo mismo con $\Delta_{1}$ y $\Delta_{2}$
Tabla 6: Elementos repetidos en el intercambio de atributos para

$\left(X_{11}+X_{12}+X_{13}=11\right)$, cuando $X_{12} \geq 3, X_{13} \geq 6$

\begin{tabular}{|c|c|c|}
\hline 0 & 3 & 8 \\
\hline 0 & 4 & 7 \\
\hline 0 & 5 & 6 \\
\hline 1 & 3 & 7 \\
\hline 1 & 4 & 6 \\
\hline 2 & 3 & 6 \\
\hline 5 & 0 & 6 \\
\hline
\end{tabular}

Fuente: Elaboración Propia

Que son elementos tanto de los conjuntos $\Delta_{1}$ y $\Delta_{2}$, de forma análoga ocurrirá en la última terna (5 0 6), que es un elemento de $\Delta y \Delta_{2}$. Por lo tanto, algunos elementos " $\mathrm{x}$ " de $\Delta$ también pertenecen al conjunto $\Delta_{1}$, es decir

$$
\begin{gathered}
x \in \Delta \cap \Delta_{1} \text { cuando } x \in \Delta, x \in \Delta_{1} \\
y \in \Delta_{1} \cap \Delta_{2} \text { cuando } y \in \Delta_{1}, y \in \Delta_{2} \\
z \in \Delta \cap \Delta_{2} \text { cuando } z \in \Delta, z \in \Delta_{2}
\end{gathered}
$$

Entonces, el conjunto $\boldsymbol{B}^{\boldsymbol{C}}$ tiene la siguiente estructura

$$
B^{C}=\Delta \cup \Delta_{1} \cup \Delta_{2}-\Delta \cap \Delta_{1} \cap \Delta_{2}
$$

Donde las intersecciones serán permutaciones de elementos de asteriscos y rayas siempre que estén fijos $\Delta, \Delta_{1} \mathrm{y} \Delta_{2}$.

Empecemos con el análisis, la intersección $\Delta \cap \Delta_{1}$, tiene la siguiente permutación

$$
\Delta\left|\Delta_{1}\right| * * *
$$

Quedando fijos $\Delta$ y $\Delta_{1}$, en la primera y segunda columna respectivamente

$$
P R_{5}^{3,2}=\frac{5 !}{3 ! \cdot 2 !}=10
$$


La intersección $\Delta_{1} \cap \Delta_{2}$, tiene la siguiente permutación

$$
* *\left|\Delta_{1}\right| \Delta_{2}
$$

Quedando fijos $\Delta_{1}, \Delta_{2}$ en la segunda y tercera columna respectivamente

$$
\begin{aligned}
& P R_{4}^{2,2}=\frac{4 !}{2 ! \cdot 2 !}=6 \\
& B^{C}=\left(P R_{8}^{6,2}+P R_{10}^{8,2}+P R_{7}^{5,2}\right)-\left(P R_{5}^{3,2}+P R_{4}^{2,2}+P R_{2}^{0,2}\right) \\
& B^{C}=(28+45+21)-(10+6+1)=77 \\
& P R_{2}^{0,2}=\frac{2 !}{0 ! \cdot 2 !}=1
\end{aligned}
$$

Ahora se reemplaza en

$$
B-A-B^{C}
$$

Tenemos

$$
B=78-77=1
$$

Finalmente se obtiene el resultado esperado $r=1$.
Finalmente, la intersección $\Delta \cap \Delta_{2}$, tiene la siguiente permutación

$$
\Delta \mid: \cdots: \Delta_{2}
$$

Quedando fijos $\Delta$ y $\Delta_{2}$, en la primera y tercera columna respectivamente

Por lo tanto, la probabilidad que un individuo incida en Pobreza Multidimensional en las tres dimensiones simultáneamente es de un 1,3\%, un probabilidad mínima, es casi imposible que el individuo sea pobre Multidimensional en las tres dimensiones.

$$
P_{3}(X, z)=\frac{1}{78}=0,013
$$

